\title{
The In Planta-Produced Extracellular Proteins ECP1 and ECP2 of Cladosporium fulvum Are Virulence Factors
}

\author{
Richard Laugé, ${ }^{1}$ Matthieu H. A. J. Joosten, ${ }^{1}$ Guido F. J. M. Van den Ackerveken, ${ }^{2}$ Henk W. J. Van den \\ Broek, ${ }^{3}$ and Pierre J. G. M. De Wit ${ }^{1}$ \\ ${ }^{1}$ Department of Phytopathology, Wageningen Agricultural University, Binnenhaven 9, 6709 PD, Wagenin- \\ gen, The Netherlands; ' Institut des Sciences Végétales, Centre National de la Recherche Scientifique, \\ 91198 Gif sur Yvette Cedex, France; ${ }^{3}$ Department of Genetics, Wageningen Agricultural University, Drei- \\ jenlaan 2, $6703 \mathrm{HA}$, Wageningen, The Netherlands \\ Received 28 February 1997. Accepted 5 May 1997.
}

The two extracellular proteins ECP1 and ECP2 are abundantly secreted by the plant-pathogenic fungus Cladosporium fulvum during colonization of the intercellular space of tomato leaves. We examined the involvement of both proteins in pathogenicity and virulence of this fungus. ECP1-deficient, ECP2-deficient, and ECP1/ECP2deficient isogenic $C$. fulvum strains were created by targeted gene replacement. Upon inoculation onto susceptible 6-week-old tomato plants, all three mutants showed reduced virulence. Deficiency in ECP2 resulted in a strain that poorly colonized the leaf tissue and secreted lower amounts of the in planta-produced ECP3, AVR4, and AVR9 proteins than the wild-type strain. The ECP2deficient strain produced little emerging mycelium and few conidia. Deficiency in ECP1 did not significantly modify colonization of the leaf tissue, but reduced secretion of in planta-produced proteins. The ECP1-deficient strain emerged from stomata of the lower epidermis, but failed to sporulate as abundantly as the wild-type strain. A strain deficient in both ECP1 and ECP2 proteins had a phenotype similar to that of the ECP2-deficient strain. Accumulation of pathogenesis-related proteins and induction of late responses, such as leaf desiccation and abscission, occurred more quickly and more severely in tomato after inoculation with the ECP1-, ECP2-, and ECP1/ECP2deficient strains than after inoculation with the wild-type strain. Moreover, partial collapse of stomatal guard cells occurred at emergence of the ECP2-deficient strain. These results indicate that the ECP1 and ECP2 proteins play a role in virulence of $C$. fulvum on tomato and suggest that both are involved in suppression of host defense responses.

The fungal pathogen Cladosporium fulvum is responsible for the leaf mold disease of tomato. During the last decade, this plant-pathogen interaction has been studied in great detail and is used as a gene-for-gene model system (Oliver 1992; De

Corresponding author: Pierre J. G. M. De Wit;

Telephone: +31-317-483130; Fax: +31-317-483412;

E-mail: Pierre.DeWit@ Medew.FYTO.WAU.NL
Wit 1995). Molecular studies have resulted in the cloning of the fungal avirulence genes $A v r 9$ (Van den Ackerveken et al. 1992) and Avr4 (Joosten et al. 1994), and the cloning of the tomato resistance genes $C f-9$ (Jones et al. 1994) and $C f-2$ (Dixon et al. 1996 ).

Little is known about the pathogenicity and virulence factors of this fungus that are responsible for its ability to colonize the apoplastic space of tomato leaves. Biochemical studies in our laboratory have shown that mannitol concentration increases drastically in the extracellular space during colonization of susceptible tomato leaves by C. fulvum (Joosten et al. 1990). Mannitol dehydrogenase, which could be involved in the nutrition of the fungus inside the leaf tissue and in pathogenesis, is currently under investigation (Noeldner et al. 1994). Mutagenesis experiments resulted in a large set of $C$. fulvum mutants that were affected in their virulence, but little information on the observed phenotypes was available (Kenyon et al. 1993).

As growth of the fungus remains restricted to the apoplastic space surrounding mesophyll cells during its whole life cycle (De Wit 1977; Lazarovits and Higgins 1976), molecules involved in communication between the fungus and the plant have been identified by analysis of apoplastic washing fluids (AF) from infected leaves (De Wit et al. 1986). This approach allowed the isolation of the two proteinaceous elicitors, AVR9 (Scholtens-Toma and De Wit 1988) and AVR4 (Joosten et al. 1994). In addition, many other low-molecular-weight proteins are present in AF, several of which are of fungal origin (De Wit et al. 1986). Two of these fungal proteins, named ECP1 and ECP2 for extracellular proteins 1 and 2, are present in abundance in AF of infected tomato leaves and have been purified (Joosten and De Wit 1988; Wubben et al. 1994). The two encoding genes, Ecp 1 and Ecp2, have been cloned (Van den Ackerveken et al. 1993). Northern (RNA) hybridization analyses performed on $C$. fulvum grown in vitro and in planta showed that the expression of both genes is highly induced during pathogenesis. Subsequent $\beta$-glucuronidase reporter studies revealed that there is expression only in the fungal mycelium growing in the apoplastic space (Wubben et al. 1994). Immunocytolocalization studies showed that, during pathogenesis, accumulation of both proteins occurs in the ex- 
tracellular matrix between the fungal hyphae and the mesophyll cells (Wubben et al. 1994). These data suggest that the ECP1 and ECP2 proteins are good candidates for pathogenicity or virulence factors of $C$. fulvum.

A strain in which the Ecp2 gene has been deleted is still able to cause disease on 2-week-old seedlings axenically grown, inoculated, and incubated in closed jars, indicating that ECP2 is dispensable for C. fulvum during pathogenesis (Marmeisse et al. 1994). We made an additional isogenic strain deficient for ECP1 and a strain deficient for both ECP1 and ECP2 by transformation-mediated gene replacement. Virulence of the three deficient strains was examined on 6week-old susceptible plants in the glasshouse, rather than on 2 -week-old seedlings in vitro, because these conditions better reflect the natural situation: C. fulvum is known to be a better leaf pathogen of adult plants than a pathogen of seedlings. In parallel, the plant defense responses induced by the ECPdeficient strains were investigated to get a better understanding of the biological functions of the ECP1 and ECP2 proteins during pathogenesis.

Here we report on a series of pathogenicity assays of mutant strains of $C$. fulvum that lack the Ecp 1 gene, the Ecp2 gene, or both genes. The assays showed that deletion of either $E c p 1, E c p 2$, or both genes significantly decreases virulence of C. fulvum on 6-week-old tomato plants.

\section{RESULTS}

\section{Generation of single and double Ecp gene replacement mutants.}

A transformation plasmid carrying a selectable marker flanked by sequences upstream and downstream of the Ecp 1 start codon was constructed. Details of the construction steps are presented in Figure 1A. The final construct carries a truncated version of the Ecp 1 gene in which both the 1.6-kb proximal sequence of the promoter and the first 21 codons of the open reading frame are lacking. A double homologous recombination event of this DNA strand at the Ecp1 locus results in replacement of the wild-type Ecp 1 sequence by a promoterless, untranslatable, truncated Ecp 1 gene as shown in Figure 1B. This construct was used for transformation of both the wild-type strain race 5 and the $\Delta E c p 2-\mathrm{D} 39$ strain in which the Ecp 2 open reading frame had already been deleted (Marmeisse et al. 1994) to create Ecp 1 and Ecp1/Ecp2 deletion mutants, respectively. Perfect gene replacement strains free of ectopic integrations of the transforming plasmid were identified either directly by Southern blot analysis on genomic DNA for the wild-type strain race 5, or by polymerase chain reaction (PCR) and subsequent Southern blot analysis for the $\Delta E c p 2-\mathrm{D} 39$ strain. Two out of 100 transformants were found to lack the Ecp 1 locus, and one of these two showed an additional illegitimate recombination event. When the 865-bp XhoI/HindIII fragment from the replacement plasmid, which contains most of the Ecp 1 open reading frame and $3^{\prime}$ sequences, was used as a probe (Fig. 1C), the exact Ecp 1 replacement transformant, designated $\Delta E c p 1-77$, exhibited a unique 1-kb XhoI replacement hybridization band and lacked the wild-type 7-kb XhoI band.

It was assumed that more transformants of the $\Delta E c p 2-\mathrm{D} 39$ strain had to be screened to obtain a strain in which both $E c p 1$ and Ecp 2 are replaced, as the promoter and terminator se- quences used in the Ecp 1 replacement marker cassette were identical to those of the Ecp2 gene replacement cassette. These sequences might therefore act as targets for homologous recombination at the Ecp2-replaced locus as well. Three hundred and fifty transformants were prescreened by PCR for absence of the wild-type Ecp 1 locus with the pair of primers indicated in Figure 1B. From the transformants that lacked the Ecp 1 locus, one double mutant, designated $\Delta E c p 1 / \Delta E c p 2-$ B94, did not show any ectopic integration (Fig. 1C).

Western blot (immunoblot) analysis of proteins present in AF isolated from tomato leaves inoculated with the $\Delta E c p 1-77$ or the $\Delta E c p 1 / \Delta E c p 2-\mathrm{B} 94$ strains confirmed that no ECP1 protein was produced by those strains (Fig. 1D) whereas $\mathrm{ECP} 3$, another fungal interaction-specific protein, was detected in both strains.

\section{Characterization of ECP-deficient strains in vitro.}

The ECP-deficient strains and the wild-type strain did not show differences in growth rate and morphology when grown either on potato dextrose agar (Fig. 2) or in B5 liquid medium (data not shown). No significant differences in sporulation rate and spore germination were observed between the mutants and the wild-type strain when grown on solid media. Southern analyses on DNA isolated from the mutant strains after several rounds of subculture did not show any changes in hybridization patterns, indicating that the Ecp-replaced strains are stable. Contour-clamped homogeneous electric field (CHEF) gel analysis of the mutant strains did not reveal changes in chromosome mobility when compared with the wild-type strain, suggesting that no significant chromosomal rearrangements had occurred (data not shown).

\section{Pathogenicity assays with the ECP-deficient strains.}

When assayed on 2-week-old tomato seedlings, the ECP1deficient strain caused normal disease symptoms, indicating no requirement of Ecpl as a pathogenicity factor for $C$. fulvum. However, this assay, in which the fungus colonizes cotyledons and primary leaves of seedlings in vitro, may not represent the natural situation, in which $C$. fulvum infects expanded leaves of mature tomato plants. Infected seedlings become desiccated after infection, whereas the upper side of infected mature leaves becomes chlorotic when the fungus initiates sporulation on the lower side of the leaf. For this reason, the ECP-deficient strains were also tested on 6-week-old tomato plants. Six-week-old plants developed chlorotic symptoms about 2 weeks after inoculation, irrespective of whether they were inoculated with the wild-type, the ECP1deficient, the ECP2-deficient, or the double ECP1/ECP2deficient strains. The double-deficient strain, which was found to be as pathogenic as the ECP2-deficient strain, was not studied in further detail.

Increase in fungal biomass during colonization of tomato leaves was estimated by light microscopy (Fig. 3) and enzyme-linked immunosorbent assays (ELISAs) (Fig. 4). In ELISAs, accumulation of three in planta-produced fungal proteins, ECP3, AVR4, and AVR9, was followed in AF from infected leaves. The ECP1-deficient strain colonized the leaf tissue as efficiently as the wild-type strain (Fig. 3A and B), while it produced less of the three in planta, extracellular proteins when compared with the wild-type strain (Fig. 4). Thus, ECP1 deficiency does not appear to severely affect the ability 
of $C$. fulvum to invade and colonize the host. The ECP2deficient strain was inhibited during development. It colonized tomato leaves poorly (Fig. 3A and $\mathrm{C}$ ) and produced low amounts of the ECP3, AVR4, and AVR9 proteins (Fig. 4).

Development of conidiophores and production of conidia were examined 20 days post inoculation by scanning electron microscopy (Fig. 5). The ECP1-deficient strain produces abundant emerging mycelium, similar to the wild-type strain. However, the major part of the leaf was covered with white, branched mycelium, creating a dense network of hyphae that do not differentiate into conidiophores (Fig. 5B), in contrast to the wild-type strain, in which hyphae do differentiate into conidiophores (Fig. 5A). The ECP2-deficient strain produced little emerging mycelium, and the greater part of the leaf sur-
A
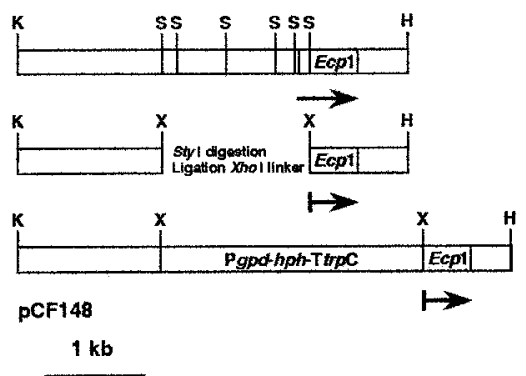

C

B
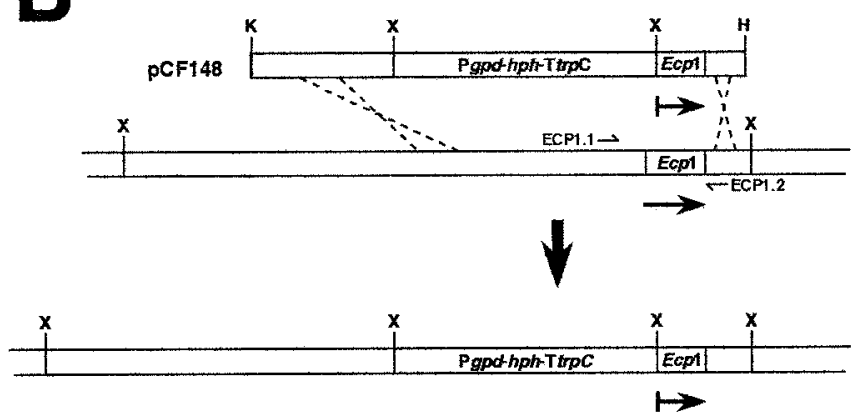

D

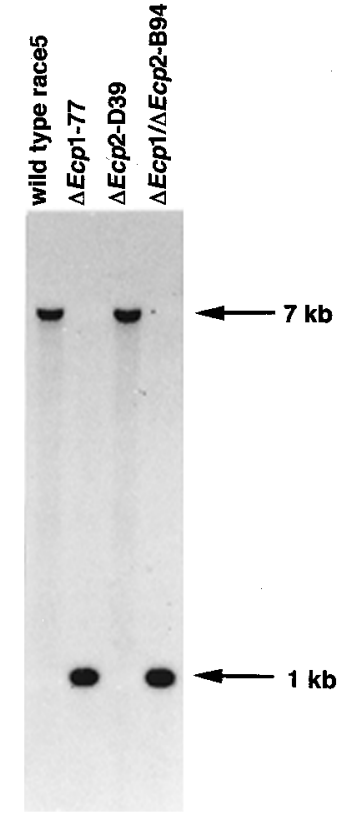

ECP1

ECP2

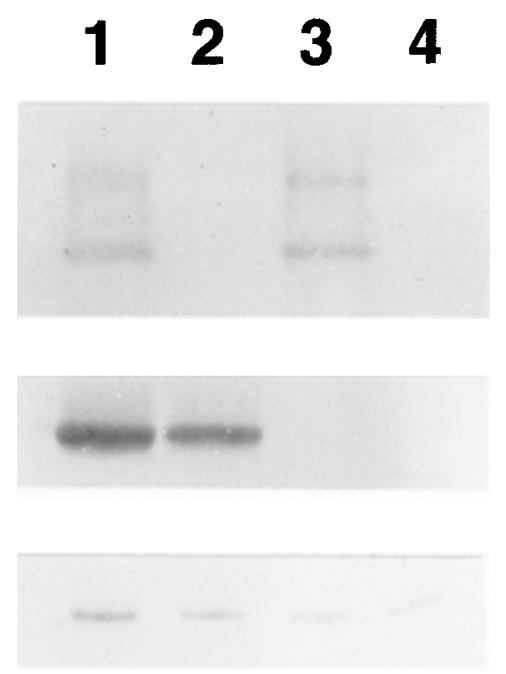

ECP3

Fig. 1. Construction and analysis of ECP1-deficient strains. A, Construction of $E c p 1$ replacement plasmid. A 4-kb subclone of $E c p 1$ gene was digested with StyI to remove a 1.5-kb fragment comprising Ecp 1 promoter and first part of the open reading frame. StyI cohesive ends were filled up by Klenow polymerase treatment; XhoI linkers were ligated. An XhoI resistance cassette consisting of the bacterial hygromycin B resistance (hph) gene under the control of the Aspergillus nidulans gpd promoter and $\operatorname{trp} \mathrm{C}$ terminator was then inserted. B, Representation of a double homologous recombination event at the Ecp 1 locus. To isolate an Ecp 1 replacement mutant of $\Delta E c p 2-\mathrm{D} 39$, transformants were screened for absence of the wild-type $E c p 1$ locus by polymerase chain reaction with ECP1.1 and ECP1.2 as primers. Restriction sites: K, KpnI; S, StyI; H, HindIII; X, XhoI. C, Southern blot analysis of the Ecp 1 locus. Genomic DNA from the wild-type race 5, the Ecp1 replacement mutant ( $\Delta E c p 1-77)$, the Ecp2 replacement mutant ( $\Delta E c p 2-D 39)$, and the double replacement mutant $(\Delta E c p 1 / \Delta E c p 2-\mathrm{B} 94)$ was digested with XhoI and probed with the 865 -bp XhoI/HindIII fragment from pCF148. D, Western blot (immunoblot) analysis of $5 \mu \mathrm{l}$ of apoplastic washing fluid (AF) isolated from infected plant material 12 days post inoculation probed with ECP1, ECP2, and ECP3 polyclonal antibodies. ECP1 was probed after blotting of a native high pH polyacrylamide electrophoresis gel in which the protein gives two signals. ECP2 and ECP3 were probed after blotting of a denaturing sodium dodecyl sulfate-polyacrylamide electrophoresis gel in which both proteins give one signal. Lane 1, wild-type race 5; lane 2, ECP1-deficient strain $\Delta E c p 1$-77; lane 3, ECP2-deficient strain $\Delta E c p 2-\mathrm{D} 39$; lane 4, ECP1/ECP2-deficient strain $\Delta E c p 1 / E c p 2-\mathrm{B} 94$. 
face was free of mycelium. Short hyphae that appear to be blocked in further development emerged from stomata. In the cases when these developed further, the extrafoliar mycelium showed abortive hyphae with short and twisted internodes (Fig. 5C).

Sporulation was quantified for the ECP1- and ECP2deficient strains and compared with the wild-type strain (Fig. 6). The wild-type strain started to sporulate 15 days post inoculation and the production of conidia increased until 20 days post inoculation, after which conidia began to disperse. The ECP1-deficient strain still produced a substantial amount of conidia but significantly less than the wild-type strain. The ECP2-deficient strain produced hardly any conidia.

\section{Mixed inoculations of wild-type and ECP-deficient strains.}

To test whether the mutant strains could be complemented by ECPs produced by the wild-type strain, we performed mixed inoculations. Conidia of the ECP1-deficient strain or the ECP2-deficient strain were mixed with conidia of the wild-type strain to a final ratio of $1: 1$ prior to inoculation of 6week-old plants. We used resistance of each ECP-deficient strain to the antibiotics hygromycin $\mathrm{B}$ and phleomycin to identify the conidia obtained from co-inoculated plants. When co-inoculated with the wild-type strain, the ECP1-deficient and ECP2-deficient strains produced 25 and $11 \%$ of the total amount of conidia, respectively, at 25 days post inoculation. Thus, upon co-inoculation, the ECP-deficient strains produce significantly more conidia than when they are inoculated on plants separately (Fig. 6). These data demonstrate that partial complementation of ECP deficiency can be achieved by coinoculation with the wild-type strain.

\section{Defense responses induced by the ECP-deficient strains.}

Accumulation of pathogenesis-related (PR) proteins in the apoplast of leaves from susceptible tomato plants was studied after challenge by the wild-type strain or the ECP-deficient strains. PR protein profiles of AF sampled at several time points after inoculation are shown in Figure 7. Upon infection by the wild-type strain, significant accumulation of PR proteins had occurred at 12 days post inoculation (Fig. 7, lane 2), and no further increase was observed at 20 days post inoculation (Fig. 7, lane 3). When plants were challenged by the

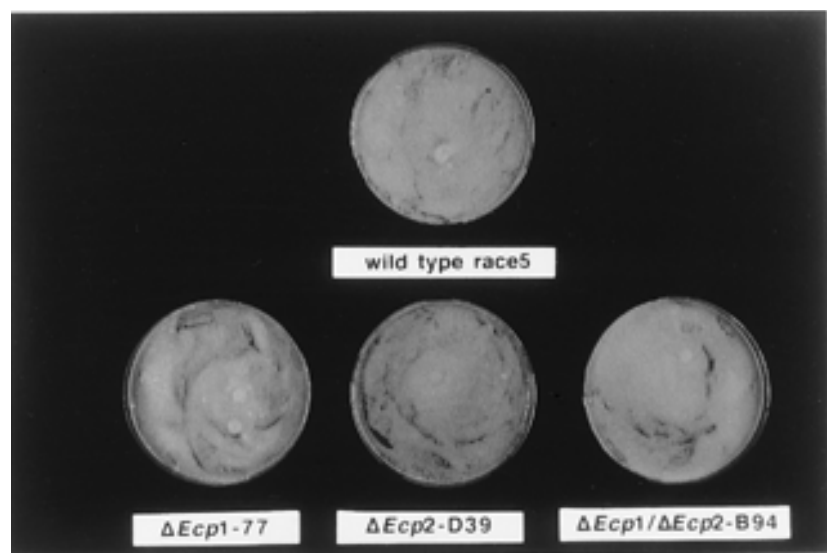

Fig. 2. Phenotype of the wild-type strain and the ECP-deficient strains cultured for 2 weeks on potato dextrose agar.
ECP1-deficient strain, no difference in accumulation of PR proteins was initially observed (Fig. 7, lane 4), while at later stages of infection accumulation of PR proteins was significantly higher than in leaves inoculated with the wild-type strain (Fig. 7, lane 5). Upon inoculation with either the ECP2deficient or the double ECP1/ECP2-deficient strains, accumulation of PR proteins in AF was found to be stronger than in leaves inoculated with the wild-type strain at both time
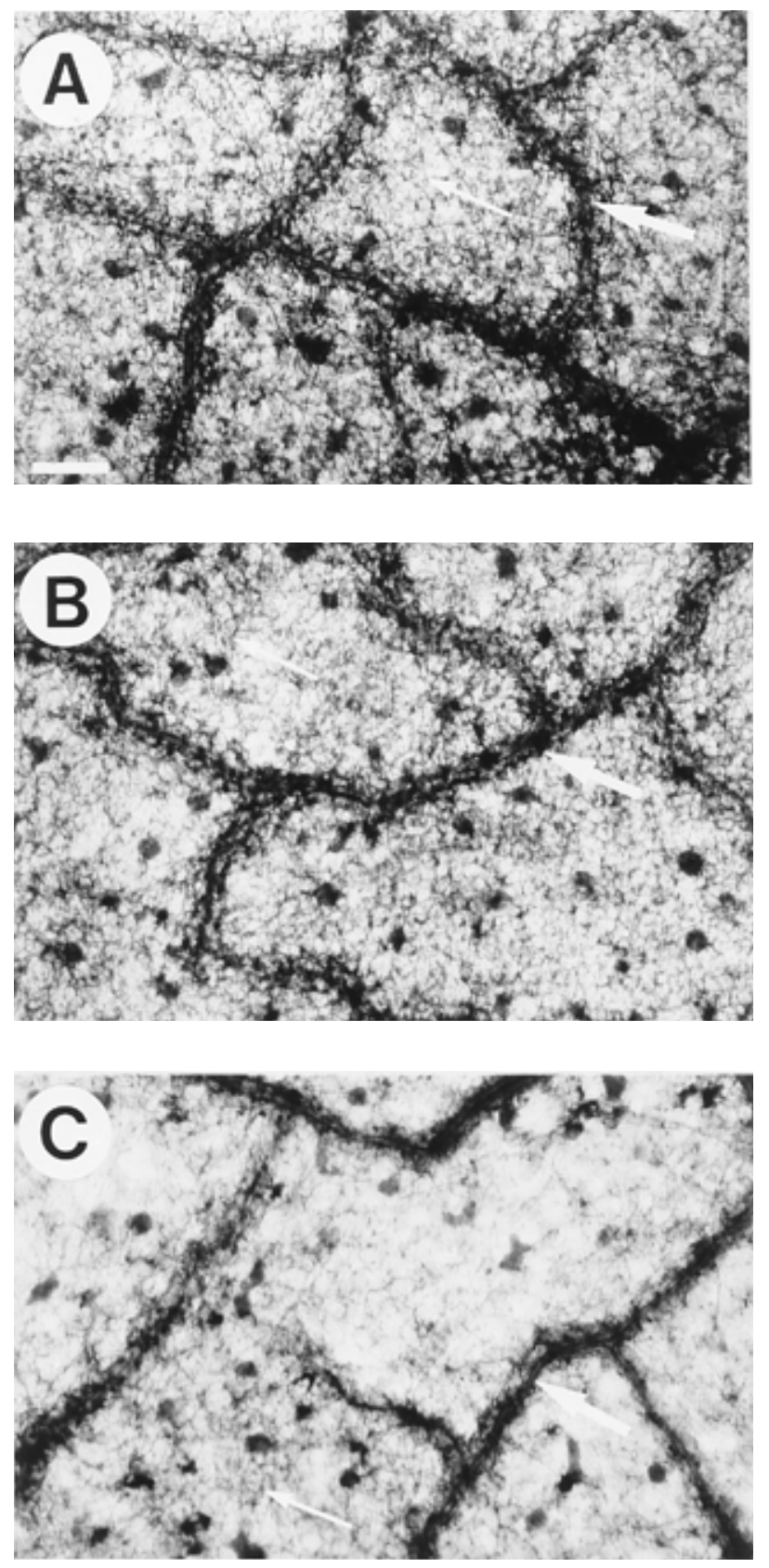

Fig. 3. Colonization of the tomato mesophyll by the wild-type and ECPdeficient strains of Cladosporium fulvum 2 weeks after inoculation. Samples were stained with cotton blue and observed by light microscopy. A, The wild type strain. B, The ECP1-deficient strain. C, The ECP2-deficient strain. Large arrows point to veins; thin arrows point to fungal mycelium. A significant decrease in staining intensity is observed for the ECP2-deficient strain $(\mathbf{C})$. Bar $=100 \mu \mathrm{m}$. 

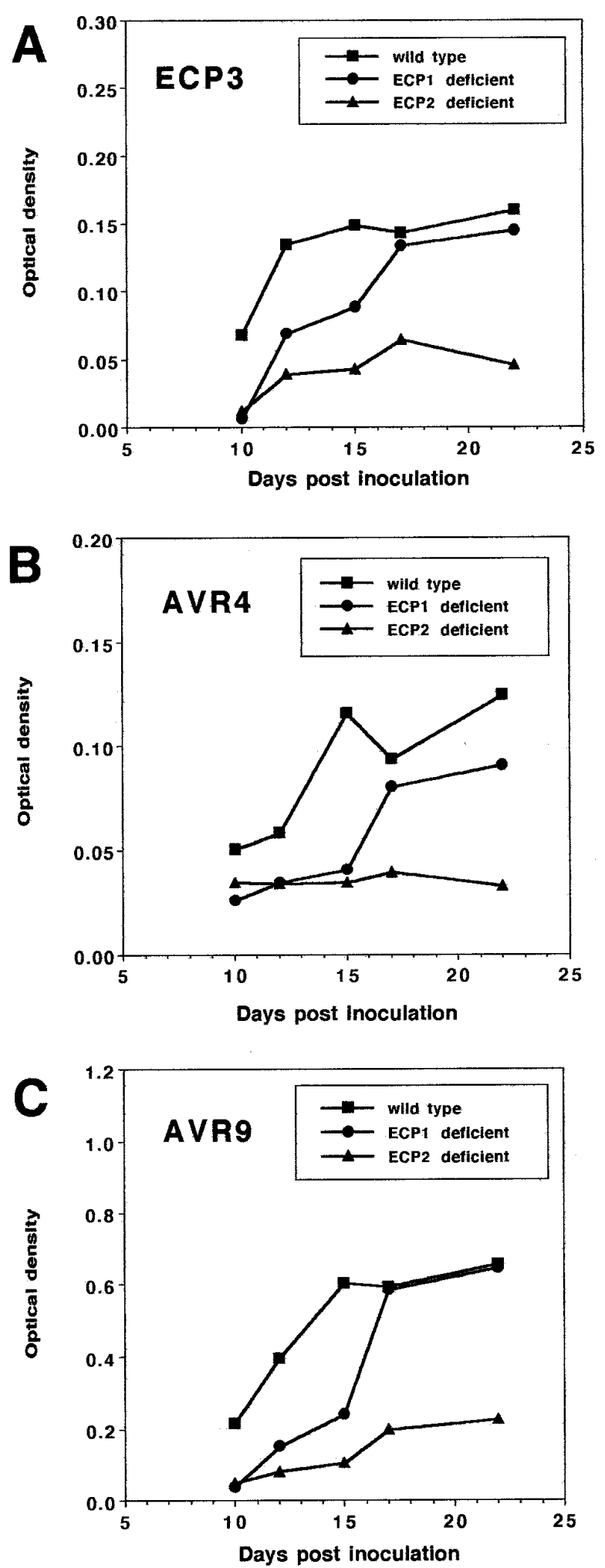

Fig. 4. Quantitative enzyme-linked immunosorbent assays on $20 \mu \mathrm{l}$ of apoplastic washing fluid isolated from tomato leaves infected by the wild-type, ECP1-, and ECP2-deficient strains at several time points after inoculation, with (A) ECP3 antibodies, (B) AVR4 antibodies, and (C) AVR9 antibodies. Optical density was determined at $405 \mathrm{~nm}$. points (Fig. 7, lanes 6, 7, 8, and 9). Thus, lack of production of either ECP1 or ECP2 resulted in additional accumulation of $\mathrm{PR}$ proteins in tomato.

During later stages of infection (4 to 5 weeks post inoculation), it was observed that wilting and abscission of leaves were more pronounced for plants inoculated with either of the ECP-deficient strains than for plants inoculated with the wildtype strain (data not shown). Collapse of stomatal guard cells at the time of emergence of mycelium through stomata was frequently observed for plants inoculated with the ECP2deficient strain (Fig. 8B). This feature was never observed in plants inoculated with the wild-type strain (Fig. 8A) or the ECP1-deficient strain (data not shown).

Virulence of the ECP-deficient strains on detached leaves.

In order to test whether the reduced virulence displayed by the ECP-deficient strains could be affected by the physiological conditions of host tissue, we did a two-step pathogenicity assay. Six-week-old plants were inoculated with conidia of the wild-type and the ECP-deficient strains. After 2 weeks, at the time when the wild-type strain was about to develop extrafoliar mycelium, infected leaflets were detached and placed at room temperature under $100 \%$ relative humidity in the dark. After incubation for one additional week, fungal development on the detached leaflets was compared with fungal development on the leaflets that had remained attached to the plant. On leaflets attached to the plant, the ECP-deficient strains remained affected in their development in comparison with the wild-type strain as described above. On the detached leaflets, all ECP-deficient strains started further development. The ECP1-deficient strain showed increased sporulation, and both the ECP2-deficient and the double ECP1/ECP2-deficient strains produced additional emerging mycelium (data not shown).

\section{DISCUSSION}

Gene replacement in $\boldsymbol{C}$. fulvum.

Gene replacement was used to test the role of the two extracellular proteins ECP1 and ECP2 in pathogenicity and virulence of $C$. fulvum. The Ecp 1 locus is surrounded by repetitive DNA that hampered the design of a replacement construct. Ecp 1 replacement could theoretically have resulted in deletion of another gene in addition to Ecp 1 . However, the results of the co-inoculation experiments led us to conclude that the complementation observed is most probably due to ECP1, and that no other important function was deleted during Ecp 1 replacement. This is supported by three observations. First, we did not observe any mutant phenotype in vitro for the ECP1deficient strain. Second, the genes encoding the extracellular proteins that are cloned so far are not clustered (R. Laugé, P. Vossen, and P. J. G. M. De Wit, unpublished). Third, we did not observe any qualitative change other than the absence of ECP1 in polyacrylamide gel electrophoresis profiles of extracellular proteins from plant material infected with the Ecp 1 mutant. We obtained Ecp 1 replacement with a frequency of ca. $1 \%$, similar to frequencies reported for Avr9 and Ecp2 (Marmeisse et al. 1993, 1994). The same technique was successfully used to obtain a double replacement mutant. The same regulatory sequences used for the hygromycin B resistance gene in the Ecp 1 replacement cassette and for the 
phleomycin resistance gene in the Ecp2 replacement cassette (Marmeisse et al. 1994) might account for the lower replacement efficiency (ca. 0.25\%) in obtaining the double Ecp 1/Ecp2 replacement mutant. Nevertheless, one double replacement mutant was recovered that showed the same stable characteristics as the single Ecp-replaced mutants. Thus, successive gene replacement is possible in the genome of $C$. fulvum and allows study of simultaneous knock-outs of several genes in the asexual fungal pathogen C. fulvum.

\section{ECP1 and ECP2 are virulence factors of $C$. fulvum.}

Disease development on 2-week-old seedlings axenically grown, inoculated, incubated in closed jars, and challenged with the ECP1-deficient strain indicated that the Ecp1 gene was not essential for pathogenicity, as had already been reported for the Ecp2 gene (Marmeisse et al. 1994). However, upon inoculation of 6-week-old plants in the glasshouse, we found a quantitative reduction in parasitic abilities of the ECP1- and ECP2-deficient strains. The ECP1-deficient strain exhibited normal growth in extracellular spaces of tomato leaves but showed decreased sporulation, whereas the ECP2deficient strain grew less abundantly inside the leaf tissue and produced little emerging mycelium. Therefore, lack of ECP1 or ECP2 during infection results in abnormal pathogenesis on tomato without, however, abolishing pathogenicity. Thus, the two ECPs can be regarded as virulence factors of $C$. fulvum. It appears essential that pathogenicity assays with $C$. fulvum are carried out under conditions that resemble natural infections.

The double ECP1/ECP2-deficient strain is still pathogenic and exhibits a phenotype similar to that of the ECP2-deficient strain. This indicates that lack of one of the two proteins cannot be compensated for by the presence of the other one, as previously hypothesized (Marmeisse et al. 1994). Each ECP must fulfill a distinct function during the interaction with tomato. Consistent with our observation that lack of ECP2 leads to a more severe decrease of virulence for $C$. fulvum than lack of ECP1, the double-deficient strain resembles the ECP2deficient strain. Subtle differences may exist between the ECP2-deficient and the double-deficient strains, but if so our pathogenicity assay was not sensitive enough to detect them.
Mixed inoculations of mutants and wild-type strains indicated that each ECP-deficient strain could only be partially complemented by ECPs produced by the wild-type strain. Mutation in ECPs could occur in nature but loss of either of the two proteins in natural populations might be counter selected for because of their lowered virulence and reproductive abilities.

\section{Possible functions of ECP1 and ECP2 in pathogenesis.}

C. fulvum does not form any specialized structures to colonize tomato leaves and does not cause detectable degradation of the plant tissue. Colonization by $C$. fulvum is restricted to the intercellular spaces, without visibly deleterious effects on the plant cells, indicating that this fungus does not require classical virulence and pathogenicity factors such as cell walldegrading enzymes and toxins. The virulence functions provided by ECP1 and ECP2 remain to be determined. On the one hand, lack of ECP1 or ECP2 production affects $C$. fulvum in planta (low sporulation for the ECP1-deficient strain, poor colonization and little emerging mycelium for the ECP2deficient strain). These effects might be due to their implication in metabolic functions such as nutrient uptake or substrate conversion. On the other hand, the ECP-deficient strains trigger stronger plant defense responses such as accumulation of extracellular PR proteins. The latter observations on the plant defense responses might point to a role in counteracting part of active plant defense responses. Possibly, lack of ECPs decreases fitness of the fungus that allows the plant to defend itself more efficiently. However, an alternative is that lack of ECPs does not allow the fungus to suppress the plant defense responses that hamper fungal development in the host when they reach a certain threshold. The latter hypothesis seems more likely, based on our observations that the ECP-deficient strains seem to fully regain pathogenic capacities, including sporulation on detached leaflets, and that they are not affected in growth and sporulation in vitro. Therefore, a role in attenuation of the plant defense responses for the ECPs during the interaction with the host is more plausible. The observation of unusually strong physiological responses such as collapse of guard cells is reminiscent of some characteristics of
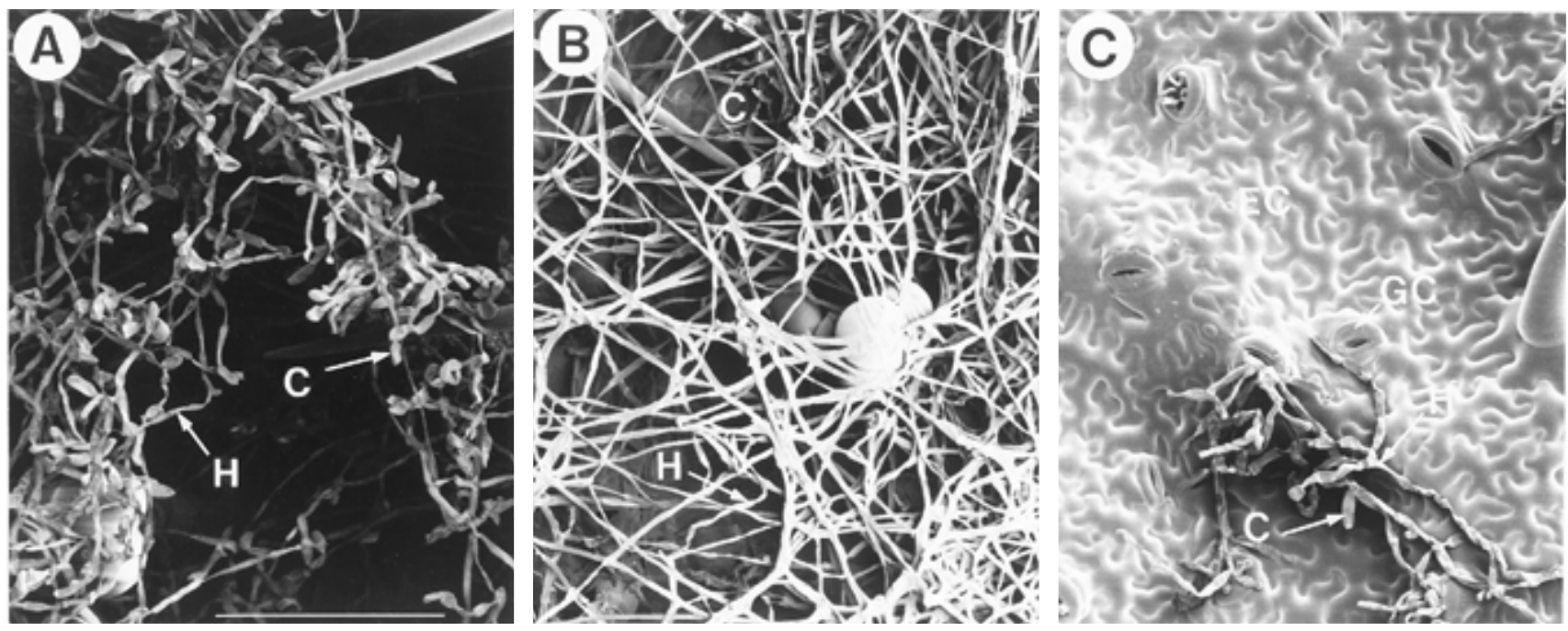

Fig. 5. Scanning-electron microscopic image of the lower side of tomato leaflets, 3 weeks after inoculation with (A) wild-type, (B) ECP1-deficient, and (C) ECP2-deficient strains. Stomatal guard cells (GC), epidermis cells (EC), fungal hyphae (H), and conidiophores (C) are indicated. Bar $=100 \mu \mathrm{m}$ 
the hypersensitive response. In immune reactions between avirulent strains of $C$. fulvum and resistant tomato genotypes, quick collapse of the host cells that come into contact with fungal hyphae is thought to be part of the successful arrest of fungal growth in early stages (Lazarovits and Higgins 1976). Similarly, rapid necrosis of stomatal guard cells has recently been proposed to be a crucial factor in resistance of Indian mustard cultivars toward Leptosphaeria maculans (Chen and Howlett 1996).

The sequence of the Ecp 1 and Ecp2 genes did not provide any clues to putative functions (Van den Ackerveken et al.

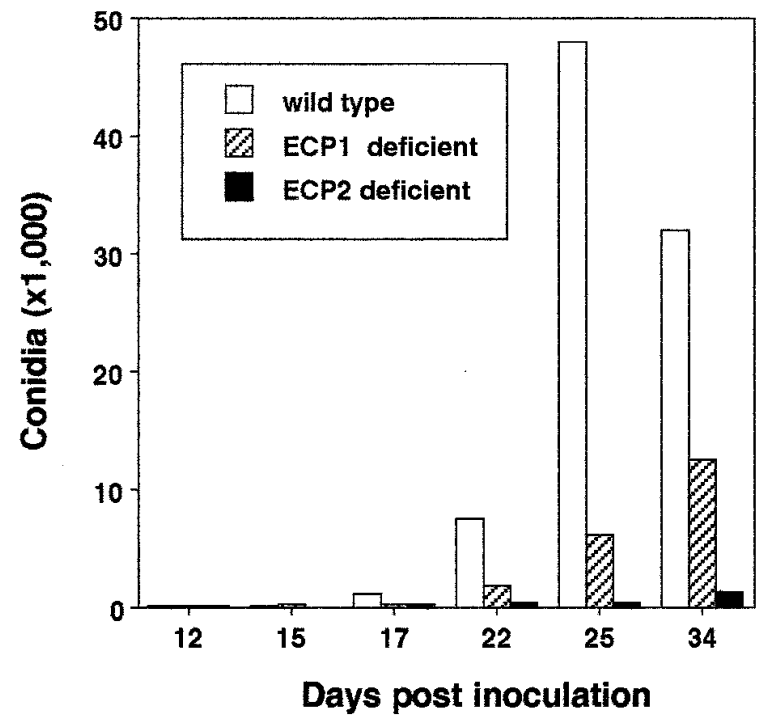

Fig. 6. Quantification of conidia isolated from six leaflets sampled from plants inoculated with wild-type, ECP1-deficient, and ECP2-deficient strains at several time points after inoculation.

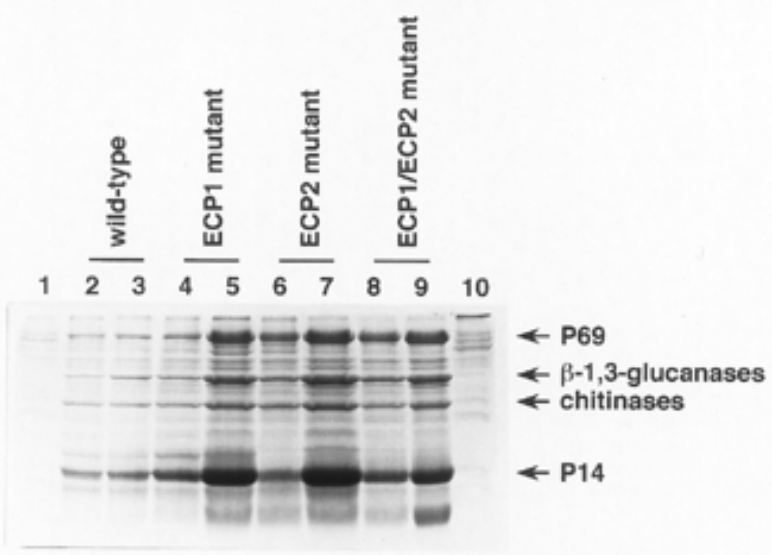

Fig. 7. Sodium dodecyl sulfate-polyacrylamide gel electrophoresis analysis of $100 \mu \mathrm{l}$ of apoplastic washing fluid (AF) isolated from plant material 12 and 20 days post inoculation, respectively. Lanes 2 and 3, wild-type race 5; lanes 4 and 5, ECP1-deficient strain; lanes 6 and 7, ECP2-deficient strain; lanes 8 and 9, ECP1/ECP2-deficient strain. Lanes 1 and 10 contain AF isolated from noninoculated plants, obtained 12 and 20 days after initiation of the experiment, respectively. The most abundant pathogenesis-related proteins that accumulate in the apoplastic space during disease development are indicated on the right side (P69, $\beta$-1,3-glucanases, chitinases, and P14).
1993). However, at the protein level, the spacing of the cysteine residues of ECP1 shows similarity to the cysteine spacing in the family of the tumor necrosis factor receptors (TNFRs) (Bazan 1993). Combined with our observations, an interesting parallel can be drawn with mammalian systems. Several mammalian viruses have been reported to produce extracellular suppressors of host defense responses (Gooding 1992). They act on cytokines, protein mediators of the immune system, a number of which are produced by host cells upon infection, including the tumor necrosis factors (TNFs). These extracellular suppressors share structural homologies with the host cell membrane-bound receptor that senses the cytokine signal. Due to this feature, they act as analogs of the host membrane-bound receptors and trap the cytokines before they reach their cellular target (Alcami and Smith, 1992; Spriggs et al. 1992). In this way, they interfere with cytokine function and disturb the establishment of host defense responses. Results of experiments carried out with viruses defi-
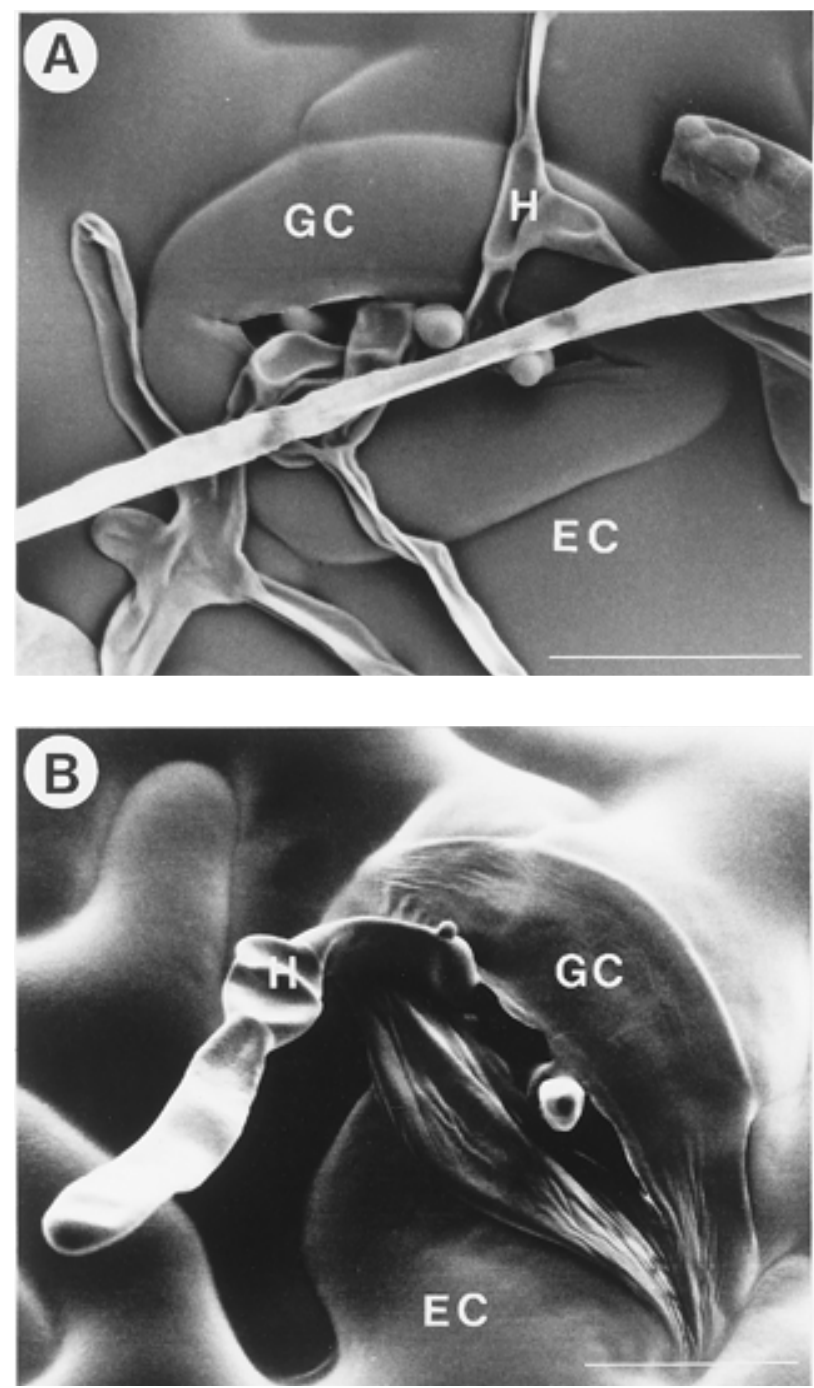

Fig. 8. Scanning-electron microscopy of stomatal guard cells at site of emergence of hyphae. Observations were made on plant material 3 weeks after inoculation with (A) wild-type and (B) ECP2-deficient strains. Stomatal guard cells (GC), epidermis cells (EC), and fungal hyphae $(\mathrm{H})$ are indicated. $\mathrm{Bar}=10 \mu \mathrm{m}$. 
cient in these suppressor proteins show striking similarities to the results we obtained with tomato plants inoculated with the ECP-deficient strains of C. fulvum. Increased host defense responses were associated with lower pathogenic abilities of the deficient viral strain (Mossman et al. 1996). Notably, one of these suppressors, the protein T2 of the Shope Fibroma virus, shares the same type of structural homology with the TNFR family as ECP1, and was shown to competitively inhibit the binding of TNF to its cell surface receptor. Testing for the existence of a homologous situation in the interaction between $C$. fulvum and tomato becomes very attractive since the recent report on the cloning of a putative plant receptor that shares structural homology with the TNFR family (Becraft et al. 1996).

\section{MATERIALS AND METHODS}

\section{Fungal strains and culture.}

Procedures for handling fungal strains were those of De Wit and Flach (1979). Strains were routinely maintained on potato dextrose agar (Merck, Darmstadt, Germany). CDA (Czapek Dox agar, Oxoid, Basingstoke, UK) containing $100 \mu \mathrm{g}$ of hygromycin B per ml (Sigma, Bornem, Belgium) was used for selection of the transformants.

\section{Fungal transformation procedure.}

Protoplasts of $C$. fulvum were prepared according to Harling et al. (1988) with the modifications of Van den Ackerveken et al. (1992). Mycelium of a 2-day-old B5 (Duchefa, Haarlem, The Netherlands) liquid culture of the recipient race 5 of $C$. fulvum was digested in MM (20 mM 2-[N-morpholino]ethanesulfonic acid, $\mathrm{pH} \mathrm{5.8,1} \mathrm{M} \mathrm{MgSO}_{4}$, containing $5 \mathrm{mg}$ of Novozym 234 [Novo Biolabs, Cambridge, UK] per ml). Chemical transformation was achieved with polyethylene glycol 6000 (Merck) according to Oliver et al. (1987). Protoplasts (1 to $5 \times 10^{6}$ ) were mixed with $10 \mu \mathrm{g}$ of the plasmid pCF148, carrying the Ecp 1 replacement cassette, in MTC ( $1 \mathrm{M} \mathrm{MgSO}_{4}$, $10 \mathrm{mM} \mathrm{CaCl}_{2}, 10 \mathrm{mM}$ Tris-HCl, $\mathrm{pH}$ 7.5). Fifteen minutes post incubation at room temperature, 1 vol of PTC ( $20 \%$ polyethylene glycol 6000, $10 \mathrm{mM} \mathrm{CaCl}_{2}, 10 \mathrm{mM}$ Tris-HCl, $\mathrm{pH} 7.5$ ) was added. After incubation for $15 \mathrm{~min}$ at room temperature, 5 vol of CM (Czapek Dox salts [Oxoid] $33.4 \mathrm{~g} /$ liter, potato dextrose broth [Difco, Detroit, MI] 12 g/liter, yeast extract [Oxoid] $1 \mathrm{~g} /$ liter, mycological peptone [Oxoid] $1 \mathrm{~g} /$ liter, casein hydrolysate [Gibco BRL, Breda, The Netherlands] $1 \mathrm{~g} /$ liter, sucrose [Merck] $273.84 \mathrm{~g} /$ liter) in top agar were added. The mixture was then plated on CM selection plates containing $100 \mu \mathrm{g}$ of hygromycin B per ml. Hygromycin B resistant transformants were obtained after 3 to 4 weeks of culture.

\section{Plants and inoculations.}

Two-week-old tomato seedlings were grown on Hoagland Agar in vitro. Six-week-old tomato plants were grown in the glasshouse in 1.5-liter pots. Both seedlings and plants were inoculated with an aqueous conidial suspension of $5 \times 10^{6}$ per $\mathrm{ml}$. The same inoculum pressure was applied for the coinoculation experiments by mixing 1 vol of a wild-type conidial suspension of $5 \times 10^{6}$ per ml with 1 vol of a mutant conidial suspension of $5 \times 10^{6}$ per ml prior to inoculation. Seedlings were briefly dipped into the solution, whereas plants were inoculated as described by De Wit (1977). Symptoms on seedlings were recorded 2 weeks after inoculation and on adult plants from 2 weeks after inoculation and onward. The inoculation experiments of seedlings and adult plants were conducted five times. Conidial inocula were checked for germination percentage on solid medium.

\section{Cloning procedures.}

All plasmid DNA manipulations were carried out essentially as described by Sambrook et al. (1989). Restriction and modifying enzymes (Gibco BRL) were used for digestions according to the manufacturer's recommendations. DNA fragments were separated on Ultrapure agarose (Gibco BRL) gels and purified with the GlassMAX DNA Isolation System (Gibco BRL).

\section{PCR on conidia.}

Putative Ecp 1 replacement mutants of the $\Delta E c p 2-D 39$ strain were prescreened by PCR, which was performed on conidia. We followed the procedure described for Magnaporthe grisea by $\mathrm{Xu}$ and Hamer (1995). The two 23-base oligonucleotides, ECP1.1 (5'-TGA GTT GCG GAA CTT CGC GGA GC-3') and ECP1.2 (5'-GTG GCA TTC AGC CTC CGC GAT GC$3^{\prime}$ ), allowed amplification of a unique 696-bp fragment for transformants that still carry a wild-type Ecp 1 locus.

DNA isolation and analyses.

Genomic DNA of C. fulvum was isolated according to Van Kan et al. (1991). Freeze-dried mycelium was ground into a fine powder and homogenized in extraction buffer $(0.5 \mathrm{M}$ $\mathrm{NaCl}, 1 \%$ sodium dodecyl sulfate (SDS), $10 \mathrm{mM}$ EDTA, 10 $\mathrm{mM}$ Tris- $\mathrm{HCl}, \mathrm{pH} 7.5)$. The mixture was extracted three times with phenol/chloroform/isoamyl alcohol (24/24/2; vol/vol/vol) and once with chloroform/isoamyl alcohol (24/1; vol/vol). The nucleic acids present in the aqueous phase were precipitated with 1 vol of isopropanol and submitted to RNAseA digestion for $30 \mathrm{~min}$. Purified DNA was obtained after repeated chloroform/isoamyl alcohol extraction, and digested with restriction enzymes. The digested DNA was separated on $0.8 \%$ agarose (Gibco BRL) TAE (0.04 M Tris-acetate, 1 mM EDTA pH 8.0) gels and transferred onto Hybond $\mathrm{N}^{+}$nylon membranes (Amersham, Buckinghamshire, UK) with a vacuum blotter (Millipore, Etten-Leur, The Netherlands). Hybridization procedures were performed overnight at $65^{\circ} \mathrm{C}$ in $5 \times$ Denhardt's reagent, $6 \times \mathrm{SSC}(1 \times \mathrm{SSC}$ is $0.15 \mathrm{M} \mathrm{NaCl}$ plus $0.015 \mathrm{M}$ sodium citrate, $\mathrm{pH} 7.0$ ), $0.5 \%$ (wt/vol) SDS. Washing steps were done at $65^{\circ} \mathrm{C}$, once for $30 \mathrm{~min}$ in $2 \times \mathrm{SSC}, 0.1 \%$ (wt/vol) SDS, and twice for $1 \mathrm{~h}$ in $0.2 \times \mathrm{SSC}, 0.1 \%$ (wt/vol) SDS. Probes were radiolabeled with the Random Primers Labeling System (Gibco BRL) and $30 \mu \mathrm{Ci} \alpha^{32} \mathrm{P}$-dATP (Amersham).

\section{Light and scanning-electron microscopy.}

All microscopy studies were performed on 1-cm-diameter leaf disks that were sampled randomly from the infected area at different time points after inoculation. For cytological examination, samples were cleared in Farmer's fluid (acetic acid/ethanol/chloroform [1/6/3; vol/vol/vol]), stained in lactophenol (lactic acid/glycerol/water [1/1/1; vol/vol/vol]) containing 30\% (wt/vol) phenol and $0.5 \%$ (wt/vol) cotton blue. After destaining in chloral hydrate $(2.5 \mathrm{~g} / \mathrm{ml})$, samples were observed by light microscopy with an Axioskop microscope (Zeiss, Oberkochen, Germany). For cryo-scanning-electron 
microscopy, samples were frozen in liquid nitrogen, sputter coated with palladium gold, and observed in a JEOL JSM 5200 (Jeol, Tokyo).

\section{Quantification of conidia production and germination of conidia.}

To quantify production of conidia, six leaflets per plant were randomly picked from a uniformly diseased area at various time points after inoculation. They were submerged in water in a 50-ml screw cap tube (Greiner, Alphen aan de Rijn, The Netherlands) and placed on a rolling bench (Greiner) for 15 min to allow the majority of the conidia to become suspended. The leaflets were discarded, and the suspended conidia were pelleted at $3,000 \times g$ and counted in a hemacytometer cell (W. Schreck, Hofheim, Germany). After the mixed inoculations, conidia were quantified in the same way. The identity of the conidia was determined by differential plating on CDA for the total amount of conidia, CDA containing 100 $\mu \mathrm{g}$ of hygromycin B per $\mathrm{ml}$ for the Ecp1-replaced strain, and CDA containing $20 \mu \mathrm{g}$ of phleomycin (Cayla, Toulouse, France) per $\mathrm{ml}$ for the Ecp2-replaced strain. As germination efficiencies of hygromycin B- and phleomycin-resistant conidia differed on media either containing the corresponding antibiotic or not, corrections were made.

\section{Isolation and analysis of apoplastic fluids.}

For the different time-course experiments, apoplastic fluids were isolated at various time points after inoculation. For each plant, six infected leaflets were randomly chosen and AF was obtained by in vacuo water infiltration followed by centrifugation at 3,000 $\times g$ according to De Wit and Spikman (1982). Protein profiles were analyzed on $15 \%$ (wt/vol) polyacrylamide slab gels under native conditions or denaturing conditions in the presence of SDS. Western blot analyses were carried out after electro transfer of the separated proteins on nitrocellulose membrane (Schleicher and Schüll, Dassel, Germany). Polyclonal antibodies against ECP1 (Joosten and De Wit, 1988), against ECP2 (Wubben et al. 1994), and against ECP3 (M. H. A. J. Joosten, R. Laugé, and P. J. G. M. De Wit, unpublished) were raised in rabbits. Polyclonal antibodies against AVR4 (Joosten et al. 1997) and against AVR9 (P. J. G. M. De Wit, P. Vossen, and M. H. A. J. Joosten, unpublished) were raised in mice. GARAP and GAMAP antibodies were obtained from Bio-Rad (Veenendaal, The Netherlands). ELISAs were performed on Nunc-Immuno Plate MaxiSorp (Nunc, Roskilde, Denmark). Optical density was read at $405 \mathrm{~nm}$ in an EL312 microplate reader (Bio-Tek, Winooski, VT).

\section{ACKNOWLEDGMENTS}

We thank Paul Vossen for assistance in the CHEF analysis of the different strains and Adriaan van Aelst (Department of Plant Cytology and Morphology, Wageningen Agricultural University) for help with the scanning-electron microscopy analysis. Paul Goodwin is acknowledged for critically reading the manuscript. This research was supported by the European Community-Human Capital and Mobility program ERBCHRXCT930244.

\section{LITERATURE CITED}

Alcami, A., and Smith, G. L. 1992. A soluble receptor for interleukin-1 $\beta$ encoded by vaccinia virus: A novel mechanism of virus modulation of the host response to infection. Cell 71:153-167.

Bazan, J. F. 1993. Emerging families of cytokines and receptors. Curr. Biol. 3:603-606.

Becraft, P. W., Stinard, P. S., and McCarty, D. R. 1996. CRINKLY4: A TNFR-like receptor kinase involved in maize epidermal differentiation. Science 273:1406-1409.

Chen, C.-Y., and Howlett, B. J. 1996. Rapid necrosis of guard cells is associated with the arrest of fungal growth in leaves of indian mustard (Brassica juncea) inoculated with avirulent isolates of Leptosphaeria maculans. Physiol. Mol. Plant Pathol. 48:73-81.

De Wit, P. J. G. M. 1977. A light and scanning-electron microscopic study of infection of tomato plants by virulent and avirulent races of Cladosporium fulvum. Neth. J. Plant Pathol. 83:109-122.

De Wit, P. J. G. M. 1995. Fungal avirulence genes and plant resistance genes: Unraveling the molecular basis of gene-for-gene interactions. Adv. Bot. Res. 21:147-185.

De Wit, P. J. G. M., Buurlage, M. B., and Hammond, K. E. 1986. The occurrence of host, pathogen and interaction-specific proteins in the apoplast of Cladosporium fulvum (syn. Fulvia fulva) infected tomato leaves. Physiol. Mol. Plant Pathol. 29:159-172.

De Wit, P. J. G. M., and Flach, W. 1979. Differential accumulation of phytoalexins in tomato leaves but not in fruits after inoculation with virulent and avirulent races of Cladosporium fulvum. Physiol. Plant Pathol. 15:257-267.

De Wit, P. J. G. M., and Spikman, G. 1982. Evidence for the occurrence of race- and cultivar-specific elicitors of necrosis in intercellular fluids of compatible interactions of Cladosporium fulvum and tomato. Physiol. Plant Pathol. 21:1-11.

Dixon, M. S., Jones, D. A., Keddie, J. S., Thomas, C. M., Harrison, K., and Jones, J. D. G. 1996. The tomato $C f-2$ disease resistance locus comprises two functional genes encoding leucine-rich repeat proteins. Cell 84:451-459

Gooding, L. R. 1992. Virus proteins that counteract host immune defenses. Cell 71:5-7.

Harling, R., Kenyon, L., Lewis, B. G., Oliver, R. P., Turner, J. G., and Coddington, A. 1988. Conditions for efficient isolation and regeneration of protoplasts from Fulvia fulva. J. Phytopathol. 122:143-146.

Jones, D. A., Thomas, C. M., Hammond-Kosack, K. E., Balint-Kurti, P. J., and Jones, J. D. G. 1994. Isolation of the tomato $C f-9$ gene for resistance to Cladosporium fulvum by transposon tagging. Science 266: 789-793.

Joosten, M. H. A. J., Cozijnsen, T. J., and De Wit, P. J. G. M. 1994. Host resistance to a fungal tomato pathogen lost by a single base-pair change in an avirulence gene. Nature 367:384-386.

Joosten, M. H. A. J., and De Wit, P. J. G. M. 1988. Isolation, purification and preliminary characterization of a protein specific for compatible Cladosporium fulvum (syn. Fulvia fulva)-tomato interactions. Physiol. Mol. Plant Pathol. 33:241-253.

Joosten, M. H. A. J., Hendrickx, L. J. M., and De Wit, P. J. G. M. 1990. Carbohydrate composition of apoplastic fluids isolated from tomato leaves inoculated with virulent or avirulent races of Cladosporium fulvum (syn. Fulvia fulva). Neth. J. Plant Pathol. 96:103-112.

Joosten, M. H. A. J., Vogelsang, R., Cozijnsen, T. J., Verbene, M. C., and De Wit, P. J. G. M. 1997. The biotrophic fungus Cladosporium fulvum circumvents $C f$-4-mediated resistance by producing instable AVR4 elicitors. Plant Cell 9:1-13.

Kenyon, L., Lewis, B. G., Coddington, A, Harling, R., and Turner, J. G. 1993. Pathogenicity mutants of the tomato leaf mould fungus Fulvia fulva (Cooke) Ciferri (syn. Cladosporium fulvum Cooke). Physiol. Mol. Plant Pathol. 43:173-191

Lazarovits, G., and Higgins, V. J. 1976. Histological comparison of Cladosporium fulvum race 1 on immune, resistant, and susceptible tomato varieties. Can. J. Bot. 54:224-234.

Marmeisse, R., Van den Ackerveken, G. F. J. M., Goosen, T., De Wit, P. J. G. M, and Van den Broek, H. W. J. 1993. Disruption of the avirulence gene $a v r 9$ in two races of the tomato pathogen Cladosporium fulvum causes virulence on tomato genotypes with the complementary resistance gene $C f 9$. Mol. Plant-Microbe Interact. 6:412-417.

Marmeisse, R., Van den Ackerveken, G. F. J. M., Goosen, T., De Wit, P. J. G. M, and Van den Broek, H. W. J. 1994. The in-planta induced ecp 2 gene of the tomato pathogen Cladosporium fulvum is not essential for pathogenicity. Curr. Genet. 26:245-250.

Mossman, K., Nation, P., Macen, J., Garbutt, M., Lucas, A., and McFadden, G. 1996. Myxoma virus M-T7, a secreted homolog of the inter- 
feron- $\gamma$ receptor, is a critical virulence factor for the development of myxomatosis in European rabbits. Virology 215:17-30.

Noeldner, P. K.-M., Coleman, M. J., Faulks, R., and Oliver, R. P. 1994. Purification and characterization of mannitol dehydrogenase from the fungal tomato pathogen Cladosporium fulvum (syn. Fulvia fulva). Physiol. Mol. Plant Pathol. 45:281-289.

Oliver, R. P. 1992. A model system for the study of plant-fungal interactions: Tomato leaf mold caused by Cladosporium fulvum. Pages 97106 in: Molecular Signals in Plant-Microbe Communications. D. P. S. Verma, ed. CRC, Boca Raton, FL.

Oliver, R. P., Roberts, I. N., Harling, R., Kenyon, L., Punt, P. J., Dingemans, M. A., and Van den Hondel, C. A. M. J. J. 1987. Transformation of Fulvia fulva, a fungal plant pathogen of tomato, to hygromycin B resistance. Curr. Genet. 12:231-233.

Sambrook, J., Fritsch, E. F., and Maniatis, T. A. 1989. Molecular Cloning: A Laboratory Manual. 2nd ed. Cold Spring Harbor Laboratory, Cold Spring Harbor, NY.

Scholtens-Toma, I. M. J., and De Wit, P. J. G. M. 1988. Purification and primary structure of a necrosis-inducing peptide from the apoplastic fluids of tomato infected with Cladosporium fulvum (syn. Fulvia fulva). Physiol. Mol. Plant Pathol. 33:59-67.

Spriggs, M., Hruby, D. E., Maliszewski, C. R., Pickup, D. J., Sims, J. E.,
Buller., R. M. L., and Van Slyke, J. 1992. Vaccinia and cowpox viruses encode a novel secreted interleukin-1-binding protein. Cell 71:145-152.

Van den Ackerveken, G. F. J. M., Van Kan, J. A. L., and De Wit, P. J. G. M. 1992. Molecular analysis of the avirulence gene avr9 of Cladosporium fulvum fully supports the gene-for-gene hypothesis. Plant J. 2:359-366.

Van den Ackerveken, G. F. J. M., Van Kan, J. A. L., Joosten, M. H. A. J., Muisers, J. M., Verbakel, H. M., and De Wit, P. J. G. M. 1993. Characterization of two putative pathogenicity genes of the fungal tomato pathogen Cladosporium fulvum. Mol. Plant-Microbe Interact. 6:210215.

Van Kan, J. A. L., Van den Ackerveken, G. F. J. M., and De Wit, P. J. G. M. 1991. Cloning and characterization of avirulence gene $a v r 9$ of the fungal pathogen Cladosporium fulvum, causal agent of tomato leaf mold. Mol. Plant-Microbe Interact. 4:52-59.

Wubben, J. P., Joosten, M. H. A. J., and De Wit, P. J. G. M. 1994. Expression and localization of two in planta induced extracellular proteins of the fungal tomato pathogen Cladosporium fulvum. Mol. Plant-Microbe Interact. 7:516-524.

Xu, J.-R., and Hamer, J. E. 1995. Assessment of Magnaporthe grisea mating type by spore PCR. Fungal Genet. Newsl. 42:80. 